\title{
Searches for new phenomena in leptonic final states using the ATLAS detector
}

\author{
Giacomo Artoni* on behalf of the ATLAS Collaboration, \\ Oxford University, $U K$ \\ E-mail: giacomo.artoniecern.ch
}

\begin{abstract}
Many theories beyond the Standard Model predict new phenomena which decay into well isolated, high-transverse-momentum leptons. Searches for new physics models with this signature are performed using the ATLAS experiment at the Large Hadron Collider (LHC). The results reported here cover searches for $W^{\prime}$ and $Z^{\prime}$ bosons using $36.1 \mathrm{fb}^{-1}$ of proton-proton collision data collected in 2015 and 2016 by the ATLAS detector at the LHC with a centre-of-mass energy of $13 \mathrm{TeV}$.
\end{abstract}

The European Physical Society Conference on High Energy Physics

5-12 July, 2017

Venice

${ }^{*}$ Speaker. 


\section{Introduction}

Many extensions of the Standard Model (SM) predict the existence of heavy spin-1 gauge bosons that could be potentially discovered at the Large Hadron Collider (LHC) [1]. These new particles, usually referred to as $W^{\prime}$ and $Z^{\prime}$, can be seen as heavier versions of the SM $W$ and $Z$ bosons, with hypothetical masses accessible by the experiments at the LHC. Assuming similar decays to those of the gauge bosons in the SM, $W^{\prime}$ and $Z^{\prime}$ might be discovered at the LHC through their decays into highly energetic, isolated leptons. The Sequential Standard Model (SSM) is a good benchmark for searches of this type as it assumes identical couplings to fermions for the $W^{\prime}$ and SM $W$ bosons. Isolated leptons with large transverse momentum $\left(p_{\mathrm{T}}\right)$ provide, in general, a clean final state signature and low backgrounds. In particular, muons offer the cleanest experimental signature and electrons provide the best energy resolution. This contribution summarises the searches performed by the ATLAS collaboration [2] using high- $p_{\mathrm{T}}$ leptons with $36.1 \mathrm{fb}^{-1}$ of data collected in 2015 and 2016 at $\sqrt{s}=13 \mathrm{TeV}$. These include a search for a $W^{\prime}$ boson [3] and searches for a $Z^{\prime}$ boson in the dielectron and dimuon final states [4] as well as in the ditau final state [5].

\section{Lepton reconstruction}

Electrons are reconstructed using the Inner Detector (ID) and the electromagnetic calorimeter. Electron candidates are clusters of energy deposits associated with ID tracks fitted with a Gaussiansum filter (GSF) to account for bremsstrahlung energy losses [6]. In order to reject background contributions from hadronic jets and photon conversions, lateral and longitudinal calorimeter shower shape, as well as tracking and cluster-track matching quantities are combined in a likelihood (LH) discriminant. Several working points (WP) are defined, depending on the desired level of signal efficiency and background rejection.

Muons are formed from tracks reconstructed in the ID and Muon Spectrometer (MS), with the combined muon track formed by a global fit using the hit information from both sub-detectors [7]. Optimal momentum resolution is achieved by requiring muon tracks to have at least three hits in each of the three precision chambers in the MS and not to traverse regions of the MS which are poorly aligned. Muon candidates are also rejected if they fall in the overlap region between the MS barrel and endcap, as well as if the MS track momentum uncertainty is too large.

Hadronic $\tau$ decays are composed of a neutrino and a set of visible decay products $\tau_{\text {had-vis, }}$, typically one or three charged pions and up to two neutral pions. The reconstruction of the visible decay products is seeded by jets and requires one or three associated tracks and an electric charge of \pm 1 [8]. A boosted decision tree (BDT) is used to further identify hadronic $\tau$ candidates and reject background from jets, combining information from calorimetric shower shapes and tracking.

\section{Search for a $W^{\prime}$ boson in the $W^{\prime} \rightarrow e v$ and $W^{\prime} \rightarrow \mu v$ decay channels}

The $W^{\prime} \rightarrow e v$ and $W^{\prime} \rightarrow \mu v$ decay channels offer the cleanest signature at the LHC for the discovery of a $W^{\prime}$ boson. As the $W^{\prime}$ is predicted to be a heavy state, the analysis uses events with one high- $p_{\mathrm{T}}$ electron or muon and large missing transverse momentum, $E_{\mathrm{T}}^{\text {miss }}$, defined as the magnitude of the negative vectorial sum of the calibrated momenta of electrons, muons and jets. 


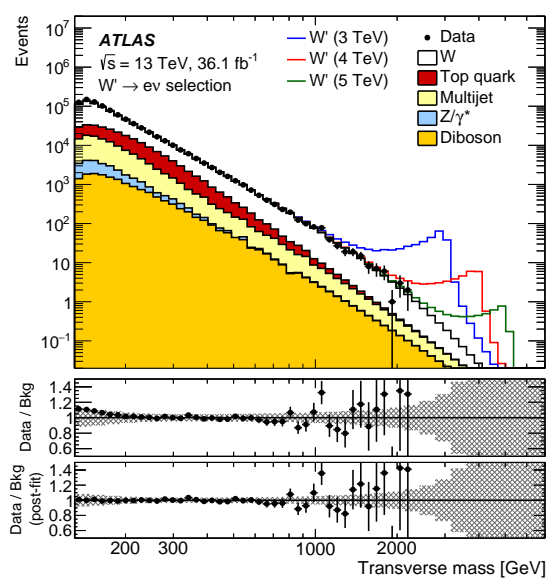

(a)

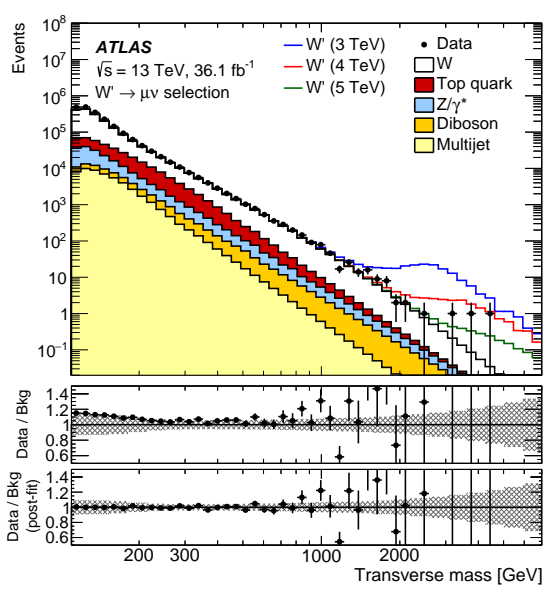

(b)

Figure 1: Transverse mass distribution (a) for the $W^{\prime} \rightarrow e v$ search and (b) for the $W^{\prime} \rightarrow \mu v$ search. Taken from Ref. [3].

A $W^{\prime}$ signal is expect to appear as an excess of events above the expected background from SM processes in the transverse mass distribution, defined as $m_{\mathrm{T}}=\sqrt{2 p_{\mathrm{T}} E_{\mathrm{T}}^{\text {miss }}(1-\cos \phi)}$, where $\phi$ represents the azimuthal angle between the $E_{\mathrm{T}}^{\mathrm{miss}}$ and electron/muon $p_{\mathrm{T}}$ directions in the transverse plane.

Events are recorded by single-electron or single-muon triggers, with additional identification criteria similar to those applied offline. Events in the electron (muon) channel are selected if the electron (muon) $p_{\mathrm{T}}$ and the missing transverse momentum both exceed the threshold of 65 (55) GeV. Tight identification criteria are applied to both electrons and muons as well as trackisolation criteria. For the electron channel, calorimeter-isolation requirements are also applied. Events are rejected if an additional loosely identified electron or muon with $p_{\mathrm{T}}>20 \mathrm{GeV}$ is found.

There are several sources of background to this analysis, most of them coming from processes with a genuine electron or muon and $E_{\mathrm{T}}^{\mathrm{miss}}$. For such processes, namely $W, t \bar{t}$ and diboson, MC simulation is used as these are well understood and modelled. In particular the dominant background comes from single $W$ production which is generated using POWHEG at next-to-leading order (NLO) in perturbative QCD (pQCD) and then normalised to next-to-next-to-leading order (NNLO) with a mass-dependent $k$-factor. A mass-dependent $k$-factor accounting for NLO electroweak (EW) effects is also employed. A minor source of background is due to events where one jet or photon is misidentified as an electron or a muon. For this background, referred to as multijet, a data-driven approach called matrix method is used [9].

The transverse mass distribution after applying all analysis selections is shown in Figure 1(a) for the electron channel and in Figure 1(b) for the muon channel, where the expected background from simulated Monte Carlo (MC) events is compared to the observed data.

Since no significant excess in the data is observed, upper limits on the cross section for the production of a $W^{\prime}$ boson times is branching ratio to one lepton generation $(\sigma \cdot B)$ are computed at $95 \%$ confidence level. When combining both channels used in this search, the observed (expected) lower mass limit for a $W^{\prime}$ boson as predicted by the SSM is 5.1 (5.2) TeV. 


\section{Search for a $Z^{\prime}$ boson in the $Z^{\prime} \rightarrow e e$ and $Z^{\prime} \rightarrow \mu \mu$ decay channels}

The $Z^{\prime}$ boson would manifest itself as a narrow resonance through its decay into a pair of leptons. Dielectron and dimuon pairs offer excellent sensitivity in this search due to their high selection efficiency and well understood backgrounds. Electrons, in particular, can offer excellent mass resolution at high energies, thus making this channel the most promising in this search.

Events in the electron channel are recorded using a two-electron-based trigger, while those in the muon channel use two different single-muon triggers with different $p_{\mathrm{T}}$ selections. Events are further selected if they feature at least two electrons or two muons, each with $p_{\mathrm{T}}>30 \mathrm{GeV}$. Only opposite-charge candidates are kept in the muon channel, while this requirement is not adopted in the electron channel due to the higher probability of misidentifying the charge of a high- $p_{\mathrm{T}}$ electron.

Similarly to the $W^{\prime}$ search, the main background components are from Drell-Yan, $t \bar{t}$ and diboson processes, which are all estimated using MC simulation. A small contribution to the background is given by multijet processes, and it is estimated from data with the same matrix method used in the previous search. This contribution is completely negligible in the case of dimuon pairs. The largest background contribution comes from Drell-Yan events, which are simulated using the NLO POWHEG event generator and then scaled with NNLO pQCD corrections and NLO EW corrections.

No significant excess of events over the dielectron (dimuon) mass spectrum is observed and an upper limit on $\sigma \cdot B$ is derived, as shown in Figure 2(a). This upper limit can be converted into lower mass limits, ranging from $3.7 \mathrm{TeV}$ to $4.5 \mathrm{TeV}$.

Limits can also be set on the coupling strength of the $Z^{\prime}$ boson relative to that of the SM $Z$ boson, denoted as $\gamma^{\prime}$. These limits are shown as a function of the $Z^{\prime}$ pole mass and of the mixing angle $\theta_{\text {Min }}$ in Figure 2(b).

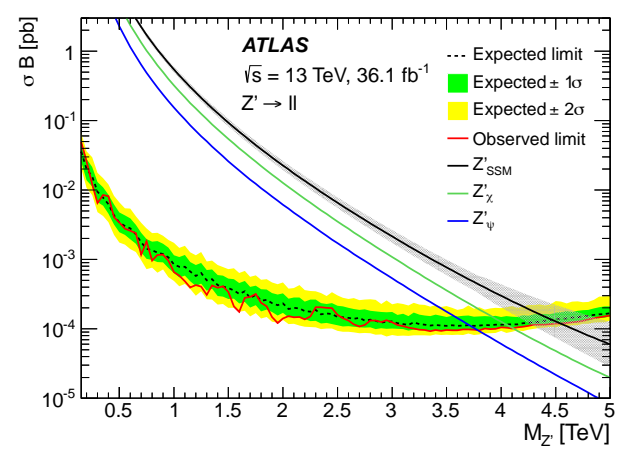

(a)

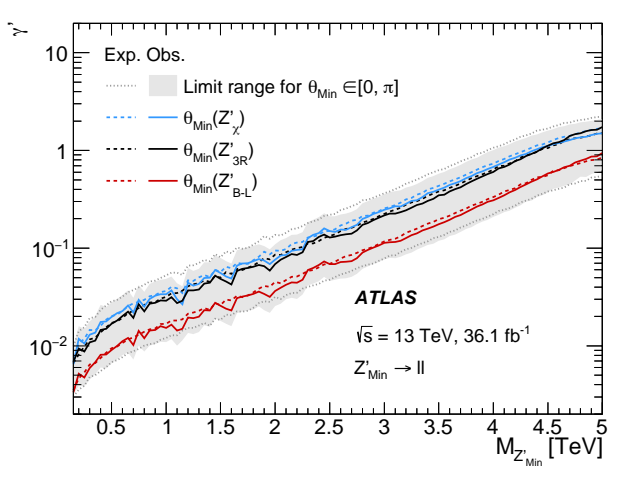

(b)

Figure 2: (a) Observed and expected upper 95\% confidence level limits on $\sigma \cdot B$ for a $Z^{\prime}$ boson as a function of the $Z^{\prime}$ pole mass. (b) Observed and expected limits at $95 \%$ confidence level on the relative coupling strength $\gamma^{\prime}$ as a function of the $Z^{\prime}$ pole mass for Minimal $Z^{\prime}$ models. Taken from Ref. [4]. 


\section{Search for a $Z^{\prime}$ boson in the $Z^{\prime} \rightarrow \tau \tau$ decay channel}

The presented search is performed in two decay modes, $\tau_{\text {lep }} \tau_{\text {had }}$ and $\tau_{\text {had }} \tau_{\text {had }}$, where $\tau_{\text {lep }}$ represents the decays of a $\tau$-lepton into an electron or a muon and neutrinos, and $\tau_{\text {had }}$ represents the decay into one or more hadrons and a neutrino.

In the $\tau_{\text {lep }} \tau_{\text {had }}$ channel, events are recorded using single-electron and single-muon triggers. These events are required to contain at least one $\tau_{\text {had-vis }}$ candidate passing the medium working point and exactly one electron or muon, with $p_{\mathrm{T}}>30 \mathrm{GeV}$ and passing medium identification as well as calorimetric and tracking isolation requirements.

Single- $\tau$ triggers are used to record events in the $\tau_{\text {had }} \tau_{\text {had }}$ channel, and the presence of two $\tau_{\text {had-vis }}$ candidates and no electron or muon is required. Both $\tau_{\text {had-vis }}$ candidates should have $p_{\mathrm{T}}>65 \mathrm{GeV}$ and the most (least) energetic one must pass medium (loose) identification criteria.

In order to optimise the separation between signal and background, the mass of the $\tau$ pair is reconstructed in both channels using the total transverse mass, $m_{\mathrm{T}}^{\text {tot }}$, defined as

$$
m_{\mathrm{T}}^{\text {tot }}=\sqrt{\left(p_{\mathrm{T}}^{\tau_{1}}+p_{\mathrm{T}}^{\tau_{2}}+E_{\mathrm{T}}^{\text {miss }}\right)^{2}-\left(\mathbf{p}_{\mathrm{T}}^{\tau_{1}}+\mathbf{p}_{\mathrm{T}}^{\tau_{2}}+\mathbf{E}_{\mathrm{T}}^{\text {miss }}\right)^{2}}
$$

where $\mathbf{p}_{\mathrm{T}}^{\tau_{1}}$ and $\mathbf{p}_{\mathrm{T}}^{\tau_{2}}$ are the momenta of the visible $\tau$ decay products projected on the transverse plane to the beam direction and $\mathbf{E}_{\mathrm{T}}^{\text {miss }}$ is the missing transverse momentum.

In the $\tau_{\text {lep }} \tau_{\text {had }}$ channel, the dominant background contribution comes from misidentified jets and it is estimated with a data-driven fake-factor technique [5]. Other contributions, where real electrons or muons are present, are estimated using MC simulation.

In the $\tau_{\text {had }} \tau_{\text {had }}$ channel the largest source of background at small values of $m_{\mathrm{T}}^{\text {tot }}$ is given by multijet production and it is estimated in a dijet control region by applying a fake-factor parametrised in $p_{\mathrm{T}}$ and track multiplicity of the $\tau_{\text {had-vis }}$. Other backgrounds are estimated from MC simulation,

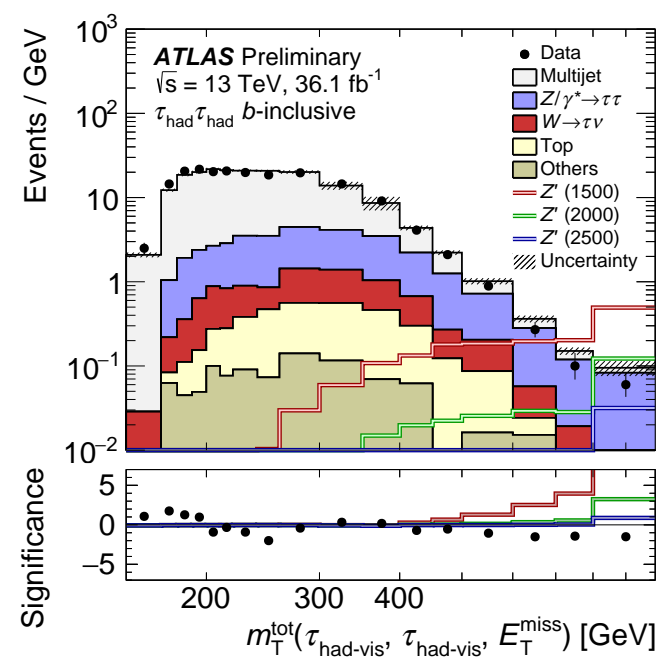

(a)

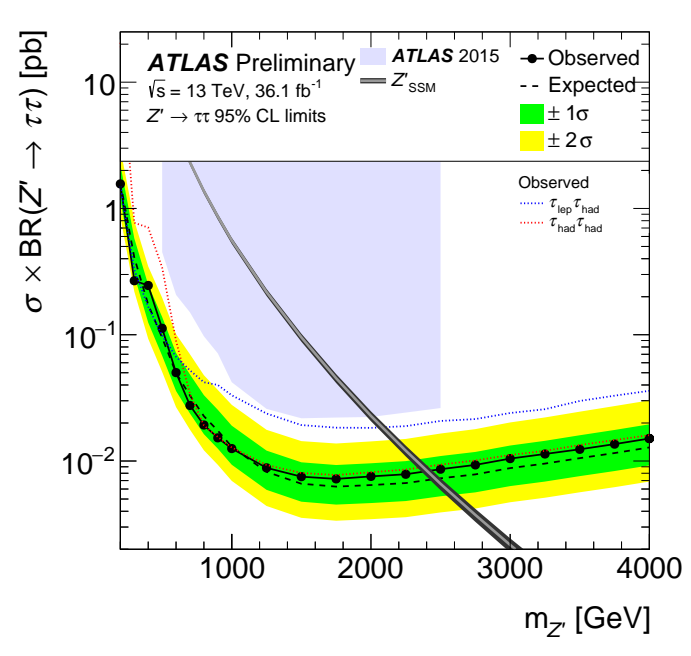

(b)

Figure 3: (a) Distribution of events as a function of $m_{\mathrm{T}}^{\text {tot }}$ in the $\tau_{\text {had }} \tau_{\text {had }}$ channel, for data and expected background. (b) Observed and expected upper limits at $95 \%$ confidence level on $\sigma \cdot B$ for a $Z^{\prime}$ boson as a function of the $Z^{\prime}$ pole mass. Taken from Ref. [5]. 
where the $\tau$ reconstruction fake rates are corrected to match those extracted from data in a $W+$ jets and a $t \bar{t}$ control regions.

When comparing the observed data to the expected background no significant excess is found, as can be seen in Figure 3(a). Upper limits on $\sigma \cdot B$ for a $Z^{\prime}$ boson are thus obtained and are shown in Figure 3(b). The $Z^{\prime} \rightarrow \tau \tau$ decay channel is particularly sensitive for a $Z^{\prime}$ boson which couples preferentially to third-generation fermions. Several models, including the Strong Flavour Model (SFM) $[10,11]$, feature this type of couplings, in the attempt of explaining the high mass of the top quark. Masses below 2.25-2.60 TeV are excluded in the context of the SFM, in the range $0.03<\sin ^{2} \phi<0.5$, where $\phi$ represents the mixing angle between the heavy and light SU(2) gauge groups.

\section{Summary}

The ATLAS experiment has performed searches for $W^{\prime}$ and $Z^{\prime}$ bosons in different scenarios using the complete data sample collected during the 2015 and 2016 data taking. Exclusion limits at $95 \%$ confidence level have been extended to $5.1 \mathrm{TeV}$ for a $W^{\prime}$ boson and up to $4.1 \mathrm{TeV}$ for a $Z^{\prime}$ boson. Masses below 2.25-2.60 TeV are also excluded by the $Z^{\prime} \rightarrow \tau \tau$ decay channel in the context of the SFM.

\section{References}

[1] L. Evans and P. Bryant (editors), LHC Machine, 2008 JINST 3 S08001

[2] ATLAS Collaboration, The ATLAS Experiment at the CERN Large Hadron Collider, 2008 JINST 3 S08003

[3] ATLAS Collaboration, Search for a new heavy gauge-boson resonance decaying into a lepton and missing transverse momentum in $36 \mathrm{fb}^{-1}$ of pp collisions at $\sqrt{s}=13 \mathrm{TeV}$ with the ATLAS experiment, arXiv:1706.04786 (2017)

[4] ATLAS Collaboration, Search for new high-mass phenomena in the dilepton final state using $36.1 \mathrm{fb}^{-1}$ of proton-proton collision data at $\sqrt{s}=13 \mathrm{TeV}$ with the ATLAS detector, arXiv:1707.02424 (2017)

[5] ATLAS Collaboration, Search for additional heavy neutral Higgs and gauge bosons in the ditau final state produced in $36.1 \mathrm{fb}^{-1}$ of pp collisions at $\sqrt{s}=13 \mathrm{TeV}$ with the ATLAS detector, ATLAS-CONF-2017-050, https://cds.cern.ch/record/2273866

[6] ATLAS Collaboration, Electron efficiency measurements with the ATLAS detector using the 2015 LHC proton-proton collision data, ATLAS-CONF-2016-024, 2016, https://cds.cern.ch/record/2157687

[7] ATLAS Collaboration, Muon reconstruction performance of the ATLAS detector in proton-proton collision data at $\sqrt{s}=13 \mathrm{TeV}$, Eur. Phys. J. C 76 (2016) 292, arXiv: 1603.05598 [hep-ex]

[8] ATLAS Collaboration, Identification and energy calibration of hadronically decaying tau leptons with the ATLAS experiment in pp collisions at $\sqrt{s}=8 \mathrm{TeV}$, Eur. Phys. J. C 75 (2015) 303, arXiv: 1412.7086 [hep-ex]

[9] ATLAS Collaboration, Search for new resonances in events with one lepton and missing transverse momentum in pp collisions at $\sqrt{s}=13 \mathrm{TeV}$ with the ATLAS detector, Phys. Lett. B 762 (2016) 334, arXiv: 1606.03977 [hep-ex] 
[10] E. Malkawi, T. Tait and C.-P. Yuan, A model of strong flavor dynamics for the top quark, Phys. Lett. B 385 (1996) 304, arXiv: hep-ph/9603349

[11] D. J. Muller and S. Nandi, Top flavor: A Separate SU(2) for the third family, Phys. Lett. B 383 (1996) 345, arXiv: hep-ph/9602390 